\title{
Novel Ablation Catheter Technology that Improves Mapping Resolution and Monitoring of Lesion Maturation
}

\author{
${ }^{1}$ ADAM PRICE, MD, 'ACHARY LESHEN, MD, ${ }^{1}$ JAMES HANSEN, MD, ${ }^{1}$ INDERPAL SINGH, MBBS, \\ ${ }^{1}$ PAWAN ARORA, MBBS, ${ }^{2}$ JOSEPH KOBLISH, BME and ${ }^{1} B O A Z$ AVITALL, MD, PhD, FHRS \\ ${ }^{1}$ University of Illinois-Chicago, Chicago, IL \\ ${ }^{2}$ Boston Scientific Corporation, Natick, MA
}

\begin{abstract}
We hypothesized that adding pin electrodes (PEs) near the ablation tip enhances the assessment of electrical activity and lesion efficacy using electrogram amplitudes (Amp) and maximum frequency spectra (Maxfreq). We used an 8-mm ablation catheter equipped with $4 \times 1$ $\mathrm{mm}$ PEs radially distributed at $90^{\circ}$ and standard recording rings. Lesions were placed under fluoroscopic and St. Jude NavX guidance to form intercaval (IC), cavotricuspid isthmus (CTI) lines and left atrial linear lesions (LL). Radiofrequency (RF) was delivered for $1 \mathrm{~min}$ at settings of $65^{\circ} \mathrm{C}$ and 65 watts. Amp and MaxFreq were recorded pre- and post ablation from bipolar PEs, PEs referenced to the $8 \mathrm{~mm}$ tip and $8 \mathrm{~mm}$ to the first ring electrode. Linear lesion gaps and atrial tachycardia were mapped and ablated using PE recordings. Post-mortem tetrazolium staining of $R F$ lesion assessment was performed. In seven dogs and 217 lesions, Amp showed marked decreases post ablation when measured with the PE compared to the 8-mm tip $(77.3 \pm 17.9 \%$ decrease versus $27.3 \pm 38.9 \%, p<0.0001)$. Using the PE, the MaxFreq decreased significantly compared with the 8$m m$ tip $56 \pm 22.9 \%$ versus $11.4 \pm 36.5, p<0.001)$. Gaps and atrial tachycardia-induced post $L L$ lesions were ablated based on PE electrical activity, indicating gaps and viable tissue. Such discrimination was not possible with the 8-mm electrode. PEs show more dramatic changes in Amp and MaxFreq versus the standard 8-mm tip. The PE enhances the detection of gaps in the linear lesion. We conclude that PEs offer significant improvement in the assessment of RF lesion efficacy and provide a useful index of lesion formation and post-ablation lesion mapping and gap detection.
\end{abstract}

ISSN 2156-3977 (print) ISSN 2156-3993 (online)

(C) 2012 Innovations in Cardiac Rhythm Management

\section{Introduction}

The introduction of large ablation tip and open irrigated ablation catheters has resulted in significant reduction in the ability of the operator to define the endpoints of lesion formation and maturation. In both these catheter technol-

Joe Koblish reports he is an employee of Boston Scientific Corporation. Dr Avitall reports he receives research funding from Boston Scientific Corporation.

This research was partially funded by: Boston Scientific Corporation.

Manuscript received November 1, 2011, final version accepted November 17, 2011.

Address correspondence to: Boaz Avitall, The University of Illinois at Chicago, Department of Medicine, Section of Cardiology, 840 South Wood Street (M/C 787), Chicago, IL 60612. E-mail: bavitall@ uic.edu ogies, temperature monitoring is a poor indicator of tissue heating since the catheter's ablation tip is cooled by either the blood flow (in the case of the $8 \mathrm{~mm}$ ) or by saline irrigation. ${ }^{1,2}$ In several publications, an amplitude reduction of the local electrogram of greater than $50 \%$ during the radiofrequency (RF) application was associated with the formation of a transmural atrial lesion. ${ }^{3-6}$ Unlike ventricular tissue, the atria consist of thin tissue with little exposure to intramyocardial blood flow or epicardial cooling from epicardial vasculature. As a result, the likelihood of extracardiac damage increases with prolonged RF application. Thus, accurate assessment of lesion maturation would reduce the risk of extracardiac injury while still maintaining ablation efficacy. With the use of an 8-mm catheter ablation tip, far-field signals reduce the ability to monitor lesion maturation via reduction in the local 
electrogram amplitude. We hypothesize that the addition of small pin electrodes in close proximity to the electrode tip on an 8-mm-tip catheter will improve the operator's ability to define effective lesion formation. In addition, pin electrodes (PEs) would also improve the ability to define conduction gaps created during the formation of linear lesions. Lastly, an effective lesion should result in a shift of the frequency spectra to lower frequencies.

\section{Methods}

All procedures were performed in compliance with the American Heart Association's guidelines for animal research and were approved by the Animal Care Committee of the University of Illinois at Chicago. Anesthesia induction with intravenous propofol $(0.5 \mathrm{mg} / \mathrm{kg})$ and maintenance with inhaled isoflurane $1.00-1.50 \%$ via endotracheal intubation was used for all invasive canine interventions. Invasive hemodynamic monitoring as well as temperature monitoring was performed throughout the entire experimental protocol and maintained at $38^{\circ} \mathrm{C}$ using a heating blanket.

\section{Ablation catheter}

The ablation catheter used was a modified 8-mm-tip catheter equipped with four PEs (Boston Scientific Corporation, Natick, MA). The PEs were $1 \mathrm{~mm}$ in diameter, radially distributed around the tip of the ablation catheter at $90^{\circ}$ orientation. The PEs were located $3 \mathrm{~mm}$ from the distal $8-\mathrm{mm}$ ablation tip, and $0.8 \mathrm{~mm}$ from one another in the circumferential orientation (Figure 1).

Recordings obtained from these catheters included: 1) bipolar recordings between the ablation tip and the first ring electrode $2.5 \mathrm{~mm}$ away from the $8 \mathrm{~mm}$ ablation electrode (tip-ring); 2) between the PEs (pin-pin); 3) between the PEs and the ablation tip (pin-tip). In addition to electrogram amplitude, maximum frequency spectra analysis was performed on each recording at the completion of the study using a Hamming Filter with a cutoff range of 0 to $250 \mathrm{~Hz}$ and 4096 samples per second. A RF notch filter was used, which was necessary to minimize electrical noise seen during RF delivery, allowing real time monitoring of the local electrograms during the ablation.

\section{Experimental and ablation protocol}

Utilizing the modified 8-mm ablation catheter, radiofrequency ablation (RFA) was performed in a temperaturecontrolled mode with the following settings: temperature $65^{\circ} \mathrm{C}$, maximum power 65 watts, and lesion duration of $1 \mathrm{~min}$. Atrial electrograms were recorded both pre- and post ablation as well as during ablation. Electrogram amplitude was visualized on the EP-Med Workmate System (St. Jude Medical, St. Paul, MN) with the filter setting of $0.5-500 \mathrm{~Hz}$.

Utilizing sterile technique, seven mongrel dogs (weight 25-35 kg) were anesthetized and underwent endotracheal intubation. Under fluoroscopic guidance, a duodecapolar catheter was inserted from the external jugular vein and positioned in the high right atrium extending into the coronary sinus. The catheter position was confirmed using EP-Med Workmate and the EnSite NaVx 3D (St. Jude Medical, St. Paul, MN) positioning system. Utilizing the 8-mm PE catheter, three-dimensional right atrial geometry was collected using the EnSite NaVx system. Under both fluoroscopic and transesophageal echocardiogram guidance, transseptal puncture was performed. Left atrial geometry was collected using the same modified ablation catheter. Pre-ablation burst pacing from the high right atrium was delivered at a cycle length of $250 \mathrm{~ms}$ for $10 \mathrm{~s}$ per burst 10 consecutive times to define the preablation atrial arrhythmia induction.

Using both three-dimensional (3D) geometry and fluoroscopic guidance, intercaval and cavotricuscpid isthmus lines were placed. In addition, left atrial linear lesions were created. Following completion of the linear lesions, mapping was performed to assess for areas of inadequate ablation as suggested by residual high frequency and atrial electrogram amplitude on bipolar recordings from the PEs of the modified ablation catheter. Post-ablation burst pacing from the high rate atrium was performed and all inducible atrial tachycardia were mapped using the PE electrograms and ablated. Post-ablation viable tissue recorded on the linear lesion line was defined as greater

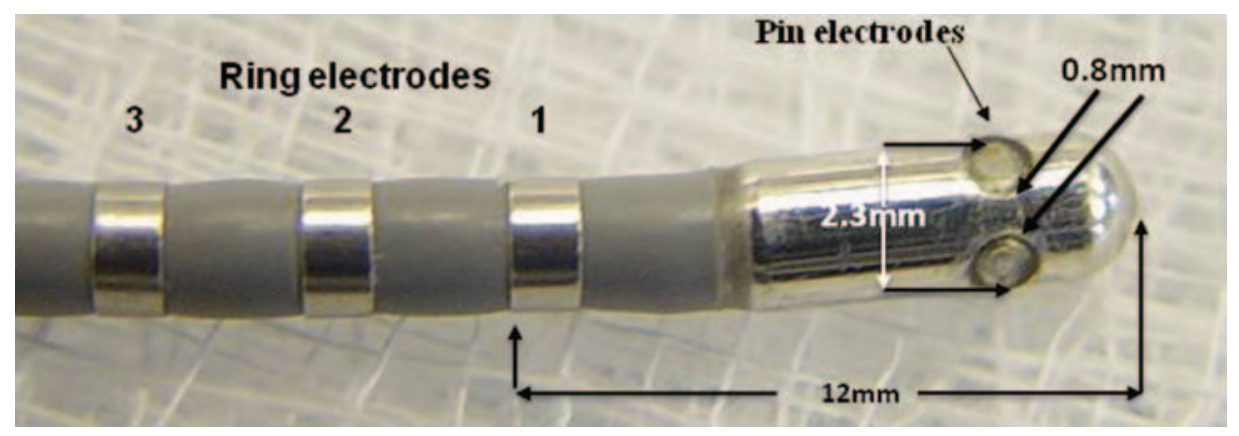

Figure 1: Modified 8-mm-tip catheter equipped with four pin electrodes (Boston Scientific Corporation, Natick, MA). The pin electrodes were $1 \mathrm{~mm}$ in diameter, radially distributed around the tip of the ablation catheter at $90^{\circ}$ orientation. The pin electrodes were located $3 \mathrm{~mm}$ from the distal $8 \mathrm{~mm}$ ablation tip, and $0.8 \mathrm{~mm}$ from one another in the circumferential orientation. 
than $0.05 \mathrm{mV}$. Post-ablation conduction block was demonstrated with activation mapping using NavX.

\section{Data collection}

During ablation, pre- and post-ablation electrogram amplitude and frequency spectra were recorded. Electrogram amplitude was measured utilizing bipolar recordings obtained from the duodecapolar and modified ablation catheter. The distal duodeca catheter was used as control to monitor electrogram amplitude stability over time. At the conclusion of the study, the hearts were excised and examined for lesions contiguity and transmurality. The hearts were soaked in tetrazolium blue stain for $8 \mathrm{~h}$. The quality of each of the individual lesions with respect to contiguity, transmurality, and char formation was reassessed. The lesions were defined as transmural and contiguous if no surviving muscle could be seen between the lesions and clear contiguous lesions could be seen on both the epicardial and endocardial surfaces.

\section{Statistical analysis}

Summary data are expressed as mean value \pm 1 SD. The paired $t$ test was used to compare continuous variables post ablation versus pre-ablation and an ANOVA test was used to compare measurements pre- and post ablation. The null hypothesis was rejected at the level of $\mathrm{p}<0.05$.

\section{Results}

In seven dogs, a total of 186 right atrial (RA) and left atrial (LA) lesions were analyzed. An additional 31 lesions were placed in the linear lesions during the ablation of atrial tachycardia and gaps. No significant differences were noted between the right atrium and left atrium in any of the parameters assessed (right atrium versus left atrium percent change of the maximum amplitude pre- versus post ablation for the tip-ring was $28.7 \pm 38.2$ versus $22.7 \pm 41.4 \mathrm{p}=\mathrm{NS}$, the maximal percent change of the pin-pin RA versus the LA $76 \pm 18.7$ versus $79.9 \pm 17.4 \mathrm{p}=\mathrm{NS}$ ); consequently the data from the RA and LA was combined. Furthermore, we have compared the recordings and ablation characteristics of the 8-mm tip with and without the PEs, and there were no differences between these catheters.

Owing to the radial orientation of the PE, analysis was performed with the PE groups stratified by greatest to least absolute amplitude change. This analysis is likely to represent the PEs arranged from the best tissue contact to the worst tissue contact. The pair with greatest amplitude and maximum frequency reduction is most likely to reflect the actual myocardial tissue contact and tissue undergoing ablation.

Table 1 summarizes the local electrogram amplitude and maximum frequency pre- and post ablation, as well as the absolute and the percentage change in the amplitude and maximum frequency comparing the tip-ring versus pin-pin versus pin-tip. Figure $\mathbf{2} \mathbf{a}, \mathbf{b}$ demonstrates the pre- and post-ablation electrogram amplitude and maximum frequency for the tip-ring, pin-pin and coronary sinus (CS) electrodes stratified by level of contact. The analysis consists of seven animals and 186 lesions placed in the left and right atria.

Figure 3 is an example a typical recordings pre- and post ablation of changes in the amplitude (a) and frequency spectra (b). As shown, the tip-ring changes were minimal for both the voltage and frequency domains (arrows a,b), whereas the recorded changes from the pin-pin were profound (arrows c,d) and no changes in the CS recordings (top two tracings).

\section{Amplitude}

As shown in Table $\mathbf{1}$ and Figure $\mathbf{2 a}$, the pre-ablation recording voltages obtained from the pin-pin are significantly larger than those recorded from the tip-ring $(4.1 \pm 2.5$ versus $1.9 \pm 1.5 \mathrm{mV}, \mathrm{p}<0.001$.). Post ablation the amplitude reduction recorded from the pin-pin is markedly greater than the tip-ring. Whereas the tip-ring reduction is $27.3 \pm 38.9 \%$, the reduction recorded from the pin-pin configuration was $77.3 \pm 17.9 \%, p<0.0001$. The recordings obtained from the pin-tip are comparable or inferior to the one obtained from the pin-pin.

The pre ablation pin-pin recordings vary significantly between level 1 versus level $4(4.1 \pm 2.5$ versus $2.1 \pm 1.6 \mathrm{mV}$, $\mathrm{p}<0.001)$ and this variation is eliminated post ablation $(0.8 \pm 0.6$ versus $0.9 \pm 0.8, \mathrm{p}=\mathrm{NS})$. The electrogram amplitude of the pin-tip was just as high pre-ablation, with even greater variation between level 1 and $4(4.5 \pm 1.8$ versus $1.6 \pm 0.9 \mathrm{mV}, \mathrm{p}<0.001)$, while post ablation significant differences persisted $(1.3 \pm 0.8$ versus $0.8 \pm 0.6 \mathrm{mV}, \mathrm{p}<$ $0.001)$. No changes were noted in the CS recordings.

\section{Frequency}

The maximum frequency (shown in Table 1 and Figure $\mathbf{3 b}$ ) exhibits similar behavior as the local electrogram amplitude. Whereas pre-ablation the tip-ring electrode maximum frequency was lower versus the pin-pin electrodes $(105 \pm 41.7$ versus $165 \pm 56.2 \mathrm{~Hz}, \mathrm{p}<0.001)$, post ablation the frequency changed little in the tip-ring with a large reduction seen in the maximum frequency in the pin-pin $(11.4 \pm 36.5$ versus $56 \pm 22.9 \%, \mathrm{p}<0.0001)$. The maximum frequency was significantly different between contact levels 1 versus 4 pre-ablation, and these differences were eliminated post ablation.

\section{Linear lesion remapping}

Data obtained during the remapping portion of the protocol are shown in Table 2 and Figure 4a,b. A total of an additional 31 lesions from four animals were included in this analysis. Similar to the initial analysis, lesions were stratified by pre-ablation amplitude to assess degree of contact and are represented as pin level 1 to 4 , with level 1 representing the best contact. Figure 4 depicts the pre- and post-ablation amplitude and maximum 
Table 1: Summary of the recorded electrogram amplitude and corresponding maximum frequency from the tip-ring, four pairs of pin to pin, and the four recordings from pin to tip pre- and post ablation, absolute change and percent change. The pin recordings are stratified based on the percent reduction in the amplitude from pre- to post ablation. Level 1 correspond to the maximal reduction likely to correspond to the pins that are in maximal contact with the tissues whereas level 4 represent the least reduction likely to represent the pin electrodes in poor or no contact with the tissues. The data provided are the average and standard deviation

\begin{tabular}{|c|c|c|c|c|c|c|c|c|c|c|c|c|}
\hline & \multicolumn{4}{|c|}{ TIP-RING } & \multicolumn{4}{|l|}{ PIN-PIN } & \multicolumn{4}{|l|}{ PIN-TIP } \\
\hline & Pre & Post & $\begin{array}{l}\text { Abs } \\
\text { Change }\end{array}$ & $\begin{array}{l}\text { Percent } \\
\text { change }\end{array}$ & Pre & Post & $\begin{array}{l}\text { Abs } \\
\text { Change }\end{array}$ & $\begin{array}{l}\text { Percent } \\
\text { change }\end{array}$ & Pre & Post & $\begin{array}{l}\text { Abs } \\
\text { Change }\end{array}$ & $\begin{array}{l}\text { Percent } \\
\text { change }\end{array}$ \\
\hline \multicolumn{13}{|c|}{ Amplitude (mV) } \\
\hline Level 1 & $\frac{1.9 \pm}{1.5}$ & $1.2 \pm 1$ & $\begin{array}{r}0.7 \pm \\
1.1\end{array}$ & $\begin{array}{r}27.3 \pm \\
38.9\end{array}$ & $\begin{array}{l}4.1 \pm \\
\quad 2.5 \\
@^{\#} \$ * \pi^{*}\end{array}$ & $\begin{array}{l}0.8 \pm \\
0.6 \\
@{ }^{\#} \$ \pi^{\mathrm{NS}}\end{array}$ & $\begin{array}{l}3.3 \pm \\
2.4 \\
@ * \$ * \pi^{*}\end{array}$ & $\begin{array}{l}77.3 \pm \\
17.9 \\
@ * \$ * \pi^{*}\end{array}$ & $\frac{4.5 \pm}{1.8 \Omega *} \pi^{*}$ & $\stackrel{1.3 \pm}{0.8 \Omega^{\mathrm{NS}} \text { * }}$ & $\begin{array}{l}3.2 \pm \\
1.9 \Omega^{*} \pi^{*}\end{array}$ & $\begin{array}{c}68.3 \pm \\
23.8 \\
\Omega^{*} \pi^{*}\end{array}$ \\
\hline Level 2 & - & - & - & - & $\begin{array}{r}3.3 \pm 1.9 \\
@ \$^{\# *}\end{array}$ & $\begin{array}{l}0.9 \pm \\
0.8 @ \# \text { \#* }\end{array}$ & $\begin{array}{l}2.4 \pm \\
1.8 \\
@^{\mathrm{NS}} \$ *\end{array}$ & $\begin{array}{c}69.4 \pm \\
19.6 \\
@^{\#} \$ *\end{array}$ & $\frac{3.4 \pm}{1.6 \Omega}$ & $\begin{array}{l}1.2 \pm \\
0.8 \Omega^{\#}\end{array}$ & $\frac{2.2 \pm}{1.6 \Omega}$ & $\begin{array}{l}61.3 \pm \\
31.3 \Omega\end{array}$ \\
\hline Level 3 & - & - & - & - & $\begin{array}{l}3.1 \pm \\
2.9 \\
@ * \$ *\end{array}$ & $\frac{1.0 \pm}{0.9 @{ }^{N S} \$ *}$ & $\begin{array}{l}2.0 \pm \\
1.9 \\
@ * \$ *\end{array}$ & $\begin{array}{l}63 \pm \\
23.9 \\
@ * \$ *\end{array}$ & $\frac{2.4 \pm}{1.3 \Omega^{\#}}$ & $\begin{array}{l}1.04 \pm \\
0.7 \Omega^{*}\end{array}$ & $1.3 \pm 1 \Omega^{*}$ & $\begin{array}{l}51.97 \pm \\
29.9 \Omega^{*}\end{array}$ \\
\hline Level 4 & - & - & - & - & $\begin{array}{l}2.1 \pm \\
1.6 \\
@ * \$^{\mathrm{NS}}\end{array}$ & $\begin{array}{l}0.9 \pm \\
0.8 @ * \$ *\end{array}$ & $\begin{array}{c}1.17 \pm \\
1.3 \\
@ * \$ *\end{array}$ & $\begin{array}{l}48.1 \pm \\
37.7 @ \# \text { \$* }\end{array}$ & $\begin{array}{l}1.6 \pm \\
0.9 \Omega *\end{array}$ & $\begin{array}{l}0.8 \pm \\
0.6 \Omega *\end{array}$ & $\begin{array}{l}0.8 \pm \\
0.9 \Omega^{\mathrm{NS}}\end{array}$ & $\begin{array}{l}41.7 \pm \\
39.1 \Omega^{*}\end{array}$ \\
\hline \multicolumn{13}{|c|}{ Frequency $(\mathrm{Hz})$} \\
\hline Level 1 & $\begin{array}{r}105.8 \pm \\
41.7\end{array}$ & $\begin{array}{r}86.9 \pm \\
36.9\end{array}$ & $\begin{array}{r}18.9 \pm \\
35.0\end{array}$ & $\begin{array}{r}11.4 \pm \\
36.5\end{array}$ & $\begin{array}{l}165.8 \pm \\
56.2 \\
@^{*} \$^{*} \pi^{*}\end{array}$ & $\begin{array}{l}65.8 \pm \\
30.6 \\
@^{\mathrm{NS}} \$ \pi^{\#}\end{array}$ & $\begin{array}{l}99.9 \pm \\
58.1 \\
@^{\#} \$^{*} \pi^{*}\end{array}$ & $\begin{array}{l}56.0 \pm \\
22.9 \\
@^{\mathrm{NS}} \$ * \pi^{*}\end{array}$ & $\begin{array}{r}143.2 \pm \\
60.7 \\
\Omega^{*} \pi^{*}\end{array}$ & $\begin{array}{l}59.8 \pm \\
34.4 \\
\Omega^{*} \pi^{\mathrm{NS}}\end{array}$ & $\begin{array}{c}84.7 \pm \\
65.9 \\
\Omega^{*} \pi^{*}\end{array}$ & $\begin{array}{l}51.5 \pm \\
32.1 \\
\Omega^{*} \pi^{*}\end{array}$ \\
\hline Level 2 & - & - & - & - & $\begin{array}{c}150.9 \pm \\
58.4 \\
@ * \$ *\end{array}$ & $\begin{array}{l}65.4 \pm \\
33.3 \\
@^{\text {NS }} \$ *\end{array}$ & $\begin{array}{c}85.5 \pm \\
57.6 \\
@^{\#} \$ *\end{array}$ & $\begin{array}{l}51.4 \pm \\
27.6 \\
@^{\mathrm{NS}} \$^{*}\end{array}$ & $\begin{array}{l}134.5 \pm \\
52.7 \Omega^{*}\end{array}$ & $\begin{array}{l}64.0 \pm \\
\quad 35.7 \Omega^{*}\end{array}$ & $\begin{array}{l}71.9 \pm \\
60.3 \Omega^{*}\end{array}$ & $\begin{array}{l}45.8 \pm \\
35.6 \Omega^{*}\end{array}$ \\
\hline Level 3 & - & - & - & - & $\begin{array}{c}130.1 \pm \\
51.1 \\
@^{\# \$ *}\end{array}$ & $\begin{array}{l}83.3 \pm \\
37.3 \\
@ * \$^{\mathrm{NS}}\end{array}$ & $\begin{array}{l}46.8 \pm \\
49.1 \\
@^{\mathrm{NS}} \$ *\end{array}$ & $\begin{array}{c}30.7 \pm \\
29.3 \\
@^{\#} \$^{*}\end{array}$ & $\begin{array}{l}114.5 \pm \\
52.78 \Omega *\end{array}$ & $\begin{array}{l}60.8 \pm \\
35.3 \Omega^{*}\end{array}$ & $\begin{array}{l}53.70 \pm \\
51.99 \Omega *\end{array}$ & $\begin{array}{l}40.4 \pm \\
33.5 \Omega^{*}\end{array}$ \\
\hline Level 4 & - & - & - & - & $\begin{array}{r}127.0 \pm \\
54.2 \\
@ * \$ *\end{array}$ & $\begin{array}{l}76.4 \pm \\
37.1 \\
@ * \$ *\end{array}$ & $\begin{array}{l}50.6 \pm \\
55.0 \\
@^{\mathrm{NS}} \$ \text { * }\end{array}$ & $\begin{array}{l}29.6 \pm \\
43.5 \\
@^{\mathrm{NS}} \$^{*}\end{array}$ & $\begin{array}{l}99.3 \pm \\
44.2 \Omega^{\mathrm{NS}}\end{array}$ & $\begin{array}{l}57.7 \pm \\
\quad 33.2 \Omega^{*}\end{array}$ & $\begin{array}{l}43.1 \pm \\
46.6 \Omega \text { * }\end{array}$ & $\begin{array}{l}36.4 \pm \\
37.1 \Omega^{*}\end{array}$ \\
\hline
\end{tabular}

@: Pin-pin versus pin-tip; \$: pin-pin versus tip-ring; $\Omega$ : pin-tip versus tip-ring; $\pi$ : level 1 versus level $4 ;{ }^{*} p<0.001,{ }^{*} p<0.05, N S$ : not significant.

frequency for the tip-ring, CS, and pin-pin electrode recordings stratified by level of contact.

\section{Gap mapping and ablation, amplitude}

When remapping for gaps, the pre ablation amplitude as measured from the pin-pin electrodes was significantly greater than the tip-ring electrodes $(2.5 \pm 1.9$ versus $0.4 \pm 0.5, \mathrm{p}<0.001)$. Post ablation, the reduction in electrogram amplitude via the pin-pin electrodes was significantly greater than the tip-ring recording. The percent change in electrogram amplitude was $97.9 \pm 8.3 \%$ for the pin-pin compared with $38.2 \pm 48 \%(\mathrm{p}<0.001)$ for the tip-ring recording. When examining the PE pairs stratified by level of tissue contact, pre ablation amplitude was significantly greater for pin level 1 compared to pin level $4(2.5 \pm 1.9$ versus $0.64 \pm 0.9, \mathrm{p}<0.001)$. These differences were eliminated post ablation (Figure 4).

\section{Gap mapping and ablation, frequency}

As shown in Table 2 and Figure 4, the pre-ablation maximum frequency recorded from the pin-pin electrodes was significantly greater than the tip-ring recordings $(148.4 \pm 54.6$ versus $81.6 \pm 35.7, \mathrm{p}<0.001)$. Post ablation, the maximum frequency decrease was greater in the PEs than in the tip-ring recordings. The percent change in maximum frequency was $83.2 \pm 17.5 \%$ versus $37 \pm 35.5 \%(\mathrm{p}<0.001)$. Examining the pin-pin recordings stratified by level of contact, pin-pin level 1 demonstrated significantly greater pre-ablation maximum frequency versus pin level $4(148.4 \pm 54.6$ versus $91.7 \pm$ $53.2 \mathrm{~Hz}, \mathrm{p}<0.001)$. Post ablation, these differences disappeared with maximum frequency between pin level 1 versus pin level $4(22.1 \pm 21.5$ versus $16.8 \pm 25.2 \mathrm{~Hz}$, $\mathrm{p}=\mathrm{NS}$ ). As noted for both the amplitude and frequency, the CS recordings remained unchanged throughout the study.

Figure 5a demonstrates the marked sensitivity of the pin-pin recording to identify viable tissues in the ablated line compared with the standard recordings from the tip-ring. During the ablation of the gap the tachycardia was terminated corresponding to the elimination of the localized electrical activity recorded from the pin-pin electrodes with little change in the tip-ring recording (Figure 5b). Post-ablation mapping using the $\mathrm{NaVx}$ system demonstrated conduction block as shown in Figure 6.

\section{Pathology}

The tetrazolium-stained lesion as shown in Figure 7 confirmed a contiguous transmural lesion. Figure 7a illustrates the lesions in the right atrium and Figure $7 \mathbf{b}$ the left atrium. No catheter char was identified during the course of ablation. 


\section{Panel A}

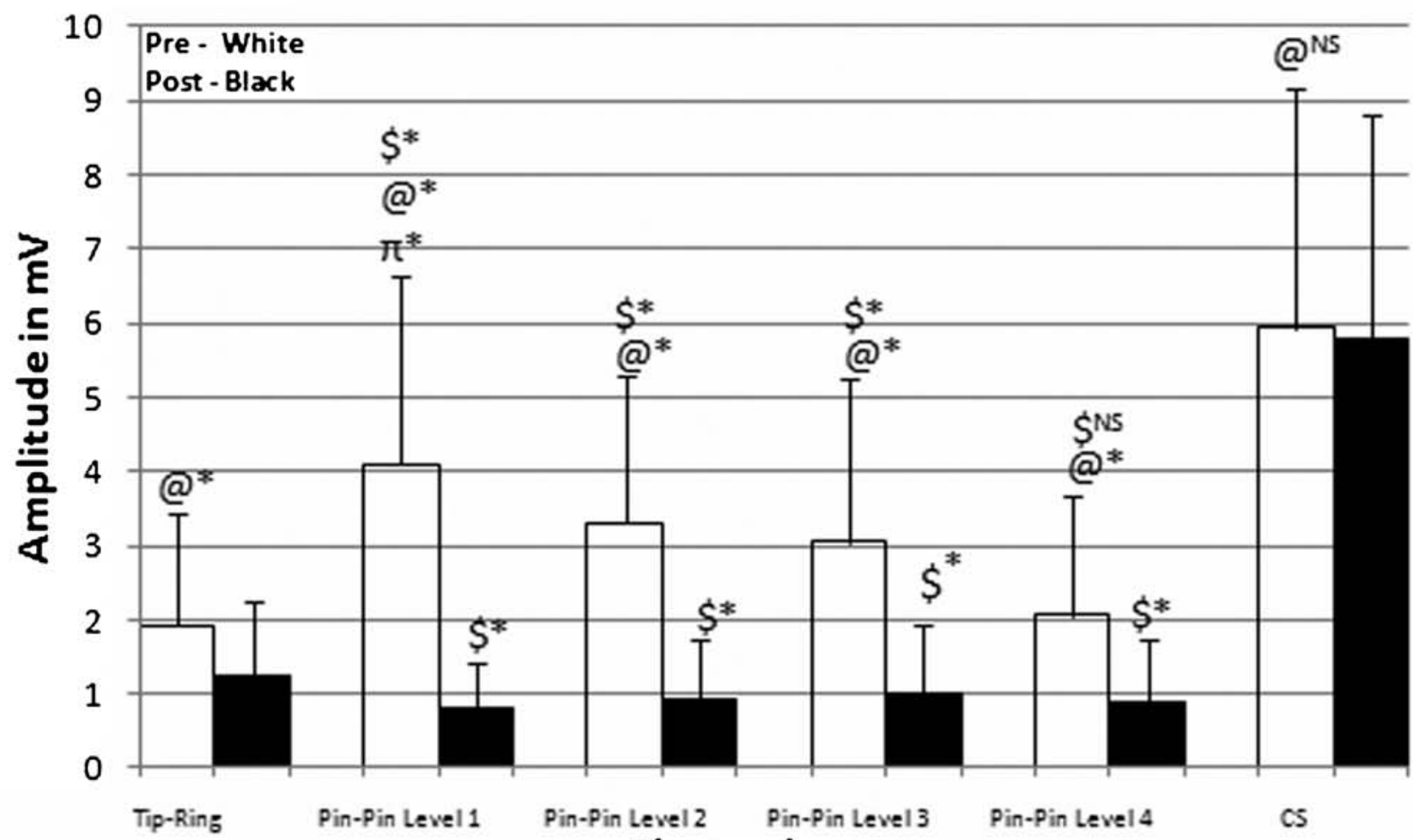

Electrodes

\section{Panel B \$*}

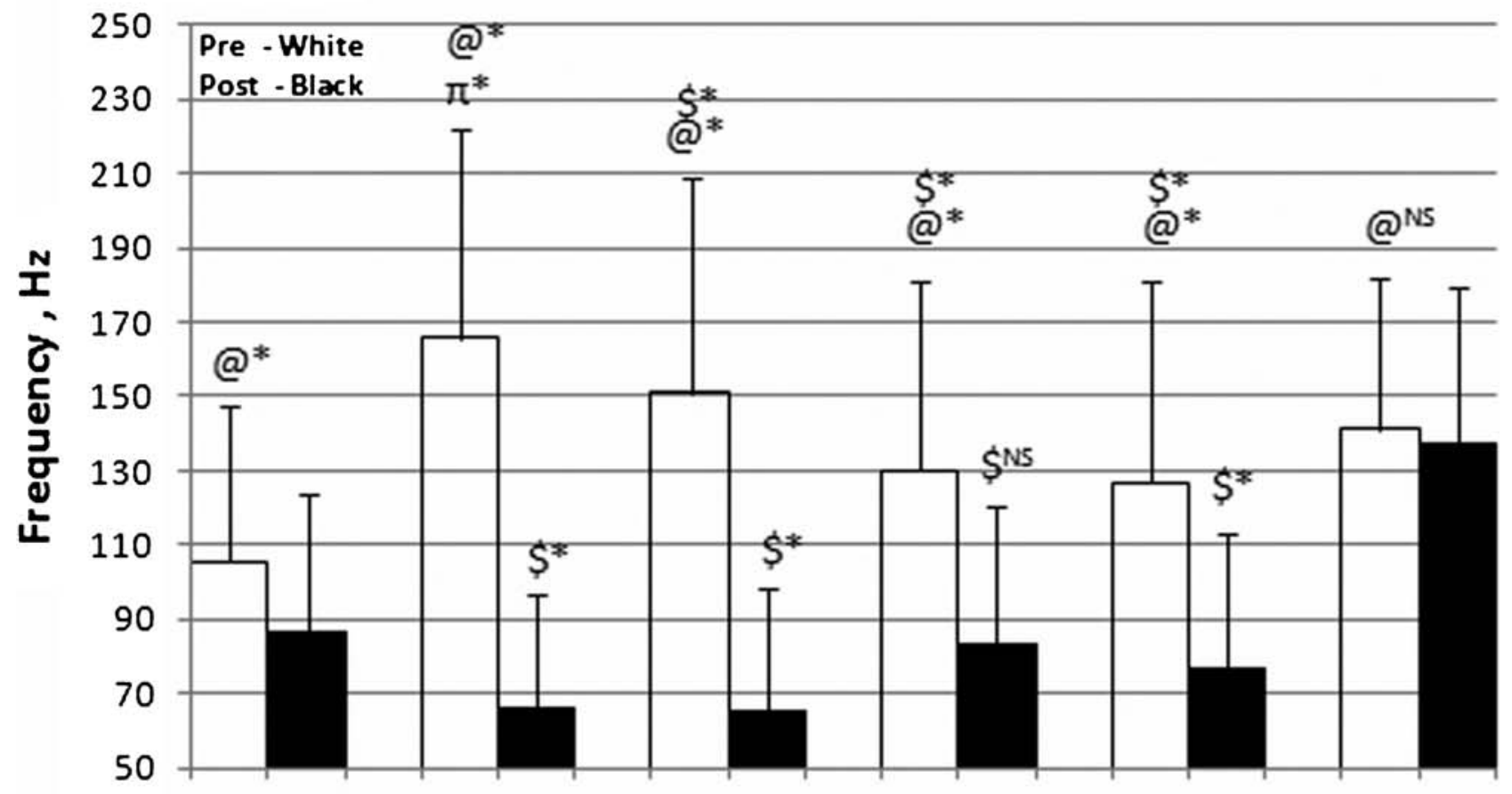

Tip-Ring Pin-Pin Level1 $\quad$ Pin-Pin Level2 Pin-Pin Level3 $\quad$ Pin-Pin Level4 $\quad$ CS

Electrodes

*p value $\$ 0.0001 @$ pre vs post; $\$$ pin-pin vs tip-ring; $\pi$ level 1 vs. level 4

\# $p$ value $\leq 0.01$

NS Non Significant 
Figure 2: The averages and SD of the electrograms amplitude and corresponding maximum frequency from the tip-ring, four pairs of pin to pin, and the four recordings from pin to tip pre- and post ablation. The pin recordings are stratified based on the percent reduction in the amplitude from pre- to post ablation. Level 1 correspond to the maximal reduction likely to correspond to the pins that are in maximal contact with the tissues whereas level 4 represent the least reduction likely to represent the pin electrodes in poor or no contact with the tissues. (a) Electrogram amplitude from seven animals and 186 lesions pre- and post ablation recorded in the right and left atria using the tip-ring, pin-pin, and distal duodeca 5-mm-apart ring electrodes positioned in the coronary sinus (CS). (b) Electrogram maximum frequency from seven animals and 186 lesions pre- and post ablation recorded in the right and left atria using the tip-ring, pin-pin, and distal duodeca 5-mm-apart ring electrodes positioned in the CS.

\section{Discussion}

The principal finding of this investigation is that the addition of four PEs circumferentially distributed $3 \mathrm{~mm}$ from the tip of the ablation electrode provides increased sensitivity to changes in the electrophysiological characteristics of the tissue subjected to RFA. The changes were recorded in the voltage and frequency domains. The marked differences in the local electrogram amplitude and frequency as well as the variation in amplitude and frequency of the local recordings between the pins are likely related to the extent of direct tissue contact of the pins with the myocardial tissue. Whereas the pins in direct tissue contact record the largest electrical amplitude and frequency, the pins that are in blood exhibit significantly lower recorded electrogram amplitude and frequency, a common characteristic of far-field recording. In contrast, the tip-ring exhibits the poorest ability to detect electrical variations associated with ablation both in the amplitude and frequency domains. The greatest changes are noted in the pin-pin electrodes that are in direct contact with the myocardium. These findings provide evidence for the utility of the PE in identifying lesion formation and maturation as well as providing discrete mapping capability. Whereas the total distance between the tip-ring (8-mm ablation electrode, $2.5-\mathrm{mm}$ tip to ring separation, and $1.5-\mathrm{mm}$ ring recording electrode) is $12 \mathrm{~mm}$, the pin-pin distance is only $0.8 \mathrm{~mm}$, which limits the far-field recording and focuses on the tissues in contact and close proximity to the electrodes.

This arrangement facilitates higher resolution mapping and ablation of arrhythmias, with improved identification of conduction gaps created during RFA.
Furthermore, the pin-pin recordings are superior to the pin to tip recordings. This finding is most likely related to the larger interelectrode distance and the incorporation of more far-field recordings when utilizing the 8-mm-tip electrode compared with the pin-pin recording.

In multiple past publications, the reduction in the local electrical activity has been proposed to be an indicator of lesion formation. ${ }^{3-5,7}$ However, these studies have utilized a 4-4.5-mm ablation tip, or multipolar electrodes of various lengths and French size. None has focused on the utility of these changes in the standard 8-mm ablation tip. It is has been shown that the 8-mm ablation electrode is a more effective ablation tool for ablation of both ventricular and atrial tissues. ${ }^{8}$ More importantly whether it is $8 \mathrm{~mm}$ (12 $\mathrm{mm}$ recording distance) or $4 \mathrm{~mm}$ (9 $\mathrm{mm}$ recording distance), this investigation for the first time defines the utility of monitoring the localized recording that is limited to the ablated tissue to define the efficacy of the ablation both in the amplitude and frequency domain.

\section{Tissue contact}

Whereas the human LV wall thickness varies from 10 to $18 \mathrm{~mm},{ }^{9}$ human left atrial thickness ranges between 1.2 and $6 \mathrm{~mm}$, with a mean of $3.7 \mathrm{~mm} \cdot{ }^{10}$ Consequently, atrial resistance to forces applied by $7-8 \mathrm{~F}$ ablation catheters result in pouching and compression of the tissue towards extracardiac structures. With increasing ablation power, excessive heating of extracardiac tissues may lead to severe and at times deadly complications, which are the consequence of the poor correlation between the ablation electrode temperature and the
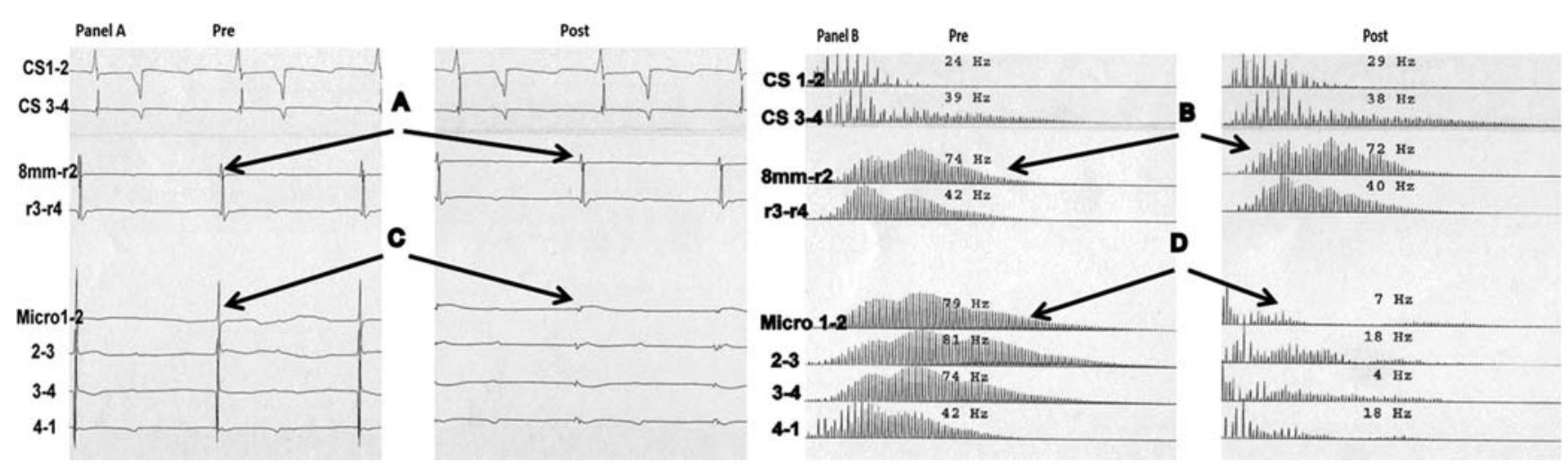

Figure 3: Changes in the voltage (a) and frequency spectra (b) pre- and post ablation. As shown, the tip-ring changes were minimal for both the voltage and frequency domains (arrows a,b) whereas the recorded changes from the pin-pin were profound (arrows $\mathrm{c}, \mathrm{d}$ ) and no changes in the coronary sinus (CS) recordings (top two tracings). 
Table 2: Linear lesions gap mapping electrogram amplitude (A) and maximum frequency (B) pre- and post-ablation, absolute change and percent change. The data is presented in the same manner as in Table 1

\begin{tabular}{|c|c|c|c|c|c|c|c|c|}
\hline & \multicolumn{4}{|l|}{ Tip-ring } & \multicolumn{4}{|l|}{ Pin-pin } \\
\hline Level 1 & $0.4 \pm 0.5$ & $0.1 \pm 0.2$ & $0.3 \pm 0.5$ & $\begin{array}{l}38.2 \pm \\
48.04\end{array}$ & $2.5 \pm 1.9 \$ * \pi^{*}$ & $0.05 \pm 0.7 \$^{\mathrm{NS}} \pi^{\mathrm{NS}}$ & $2.45 \pm 1.9 \$ * \pi^{*}$ & $97.9 \pm 8.3 \$ * \pi^{*}$ \\
\hline Level 2 & & & & & $2.1 \pm 1.6 \$ *$ & $0.03 \pm 0.12 \$^{\mathrm{NS}}$ & $2.06 \pm 1.6 \$ *$ & $97.5 \pm 10.2$ \$* \\
\hline LEVEL 1 & $81.6 \pm 35.7$ & $49.1 \pm 37.4$ & $32.5 \pm 35$ & $37 \pm 35.5$ & $\begin{array}{l}148.4 \pm \\
54.6 \${ }^{*} \pi^{*}\end{array}$ & $\begin{array}{l}22.1 \pm \\
21.5 \$^{\#} \pi^{\mathrm{NS}}\end{array}$ & $\begin{array}{l}126.3 \pm \\
59.1 \$^{*} \pi^{*}\end{array}$ & $\begin{array}{l}83.2 \pm \\
17.5 \$ * \pi^{\#}\end{array}$ \\
\hline LEVEL 2 & & & & & $143.5 \pm 60 \$ *$ & $18.1 \pm 21 \$ *$ & $125.4 \pm 66.6 \$$ * & $81.3 \pm 31.4 \$ *$ \\
\hline LEVEL 3 & & & & & $111 \pm 54 \$$ & $22.6 \pm 40 \$ \#$ & $89 \pm 62.4 \$ *$ & $77.3 \pm 35.6 \$ *$ \\
\hline LEVEL 4 & & & & & $91.7 \pm 53.2 \$^{\mathrm{NS}}$ & $16.8 \pm 25.2 \$ *$ & $74.9 \pm 56.7 \$ *$ & $70 \pm 36.2 \$ *$ \\
\hline
\end{tabular}

\$: Pin-pin versus tip-ring; $\pi$ : level 1 versus level $4 ;{ }^{*} p<0.001 ;{ }^{*} p<0.05$, NS: not significant.

tissue temperature. ${ }^{1,11-14}$ As such, avoiding extracardiac injury caused by atrial RF ablation lesions and the success of ablation depends on accurate information regarding lesion formation. Since the ultimate goal of the ablation is the creation of electrically silent tissues, we have focused on providing the operator with higher resolution electrical activity of the tissue being ablated. Currently, additional studies are being done to assess whether the maximal reduction of the atrial electrogram in the PEs identifies the completion of the lesion. Successfully titrating ablation to electrogram amplitude diminution would allow for decreased ablation time and potentially increase safety by limiting the amount of energy applied to the myocardium.

Given the atrial anatomy, limited atrial tissue thickness, and the compression of the tissues by the ablation catheter, the majority of the far-field recording is not related to the tissue thickness but rather from the surrounding tissues. The maximum frequency recorded from the PEs with the best tissue contact decreased from $165.8 \pm 56.2 \mathrm{~Hz}$ to $65.8 \pm 30.6 \mathrm{~Hz}$ following ablation. This is a result of the marked reduction in amplitude and the emergence of lower frequency spectra likely associated with current of injury that replaces the fast depolarization wave front that transverses the tissue prior to the ablation. Both the reduction of the local electrogram amplitude and the frequency shift are uniquely associated with atrial lesion formation while recording from the PEs. These changes were associated with lesion formation and a surrogate of tissue contact.

\section{Catheter to tissue contact angle}

The variation in the pin-pin electrogram amplitude and maximum frequency pre- and post ablation is likely related to the catheter to tissue orientation. Whereas the pins in direct contact with the myocardium exhibit significantly higher amplitude and maximum frequency than the pins that are in contact with the blood, these differences were eliminated post ablation, providing further indication of lesion formation.

\section{Electrical discrimination}

In a recent investigation, ${ }^{15} \mathrm{PE}$ technology was also found to provide focused recordings and improved spatial discrimination compared to the tip-ring recordings. Similarly, this investigation demonstrated the ability to map the linear lesions for gaps associated with the induction of atrial tachyarrhythmias. The technology allowed the differentiation of ablated versus electrically viable tissues, that once ablated terminated the atrial arrhythmias. In two of our seven subjects, atrial tachyarrhythmias were induced post ablation. As part of the experimental protocol, detailed mapping of the linear lesions lines were performed. As demonstrated in Figure 5, obtained during the presence of a tachyarrhythmia recorded following intercaval lesion formation, high-frequency recordings are evident on the $\mathrm{PE}$ recordings while the tip-ring recording fails to record the same high-frequency signal. Ablation at this site resulted in termination of the tachyarrhythmia. This termination occurred despite the lack of change in the tip-ring recordings. This provides further support of the improved mapping capabilities of the PEs, and demonstrates a clear utility of this catheter in identifying conduction gaps that may be present in the process of creating linear lesions.

\section{Limitations}

The electrophysiological changes post ablation were investigated in normal atrial tissues in sinus rhythm. Further studies are needed to assess the utility of this technology in diseased and fibrillating atria. Additionally, the current experimental model prohibited the assessment of individual atrial lesions due to the creation of linear 


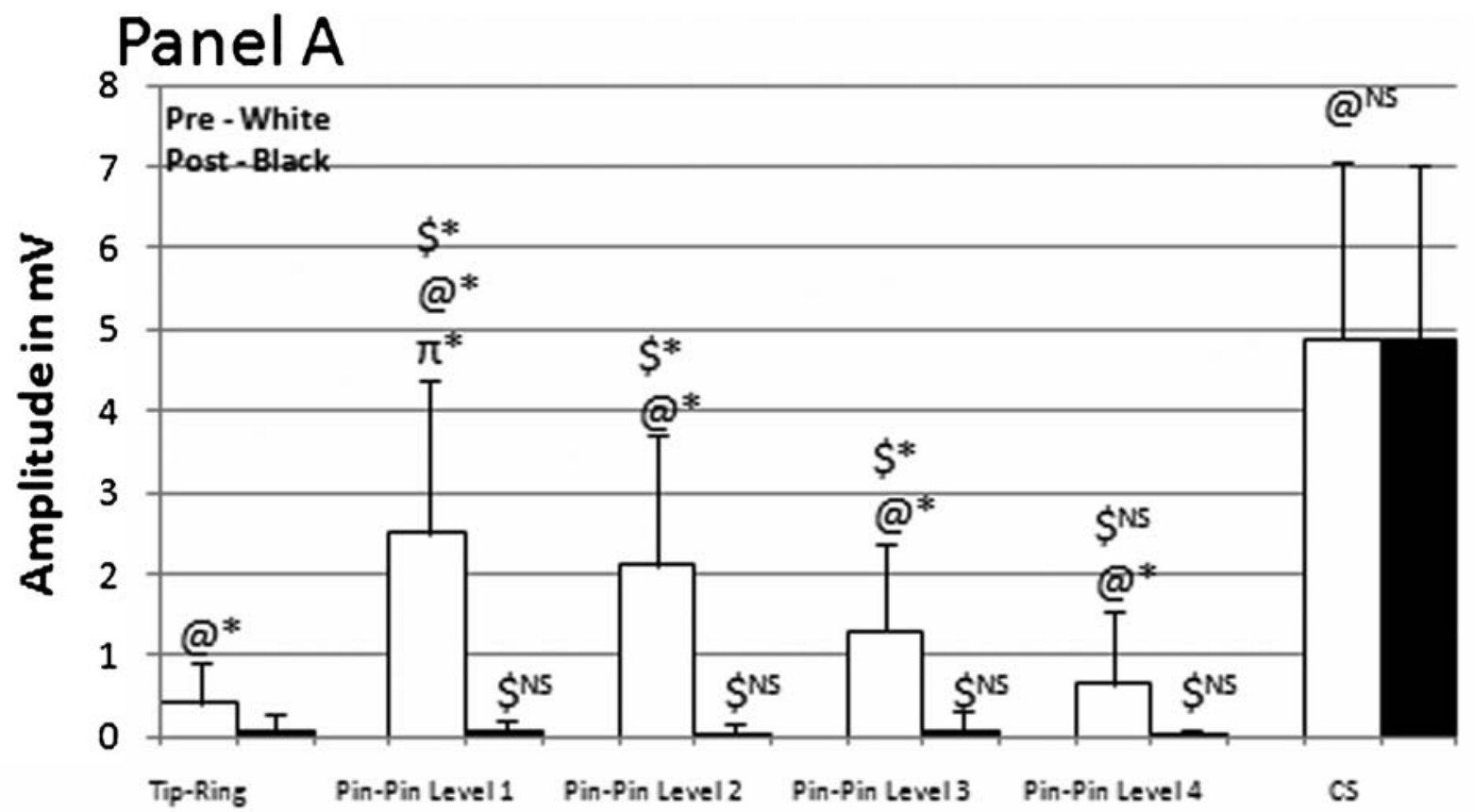

\section{Electrodes}

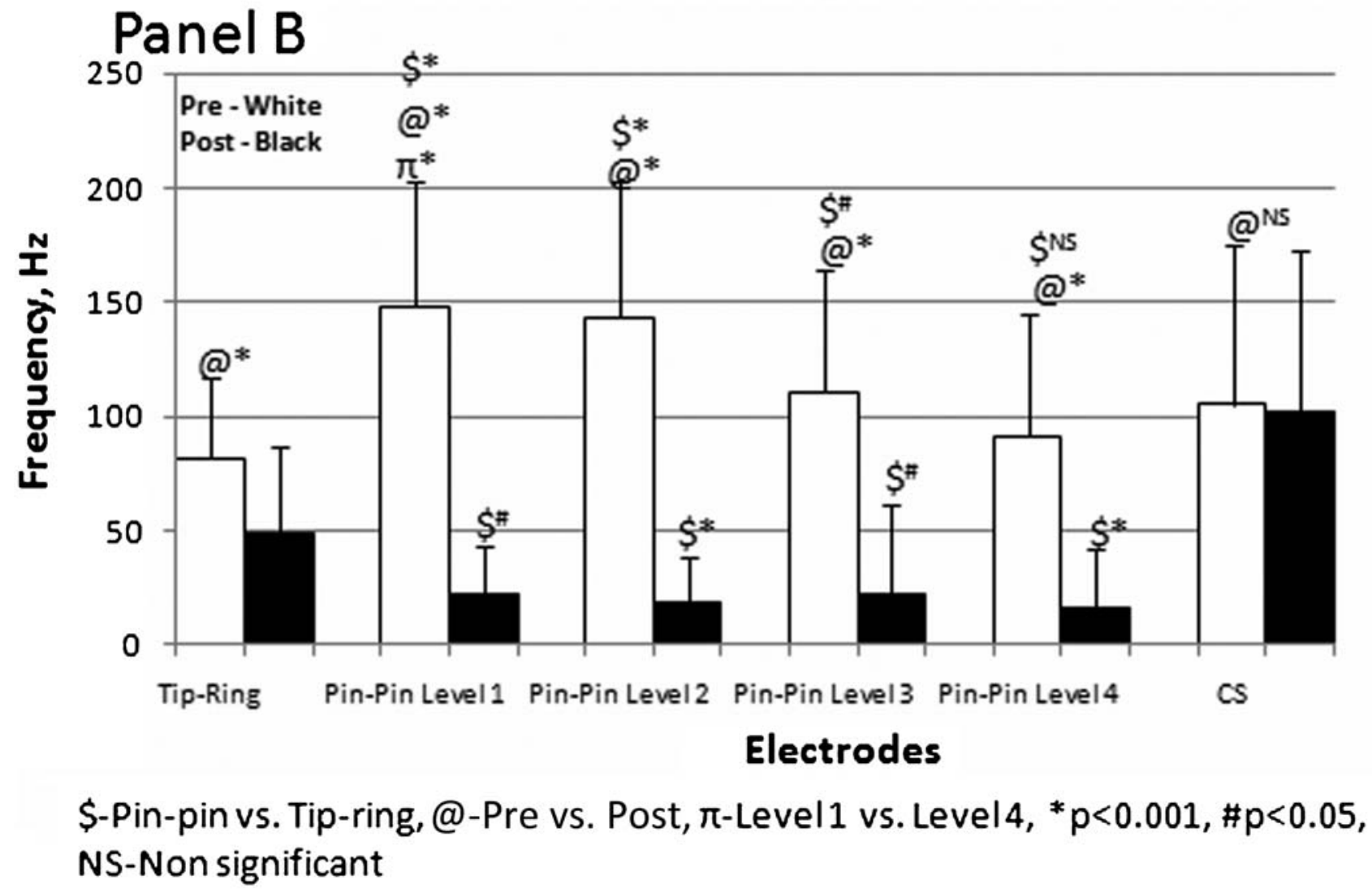

Figure 4: Gap electrogram amplitude (a) and maximum frequency (b) from four animals and 31 lesions pre- and post ablation recorded in the right and left atria using the tip-ring, pin-pin, and distal duodeca 5-mm-apart ring electrodes positioned in the CS. The averages and SD of the electrogram amplitude and corresponding maximum frequency from the tip-ring, four pairs of pin to pin, and the four recordings from pin to tip pre and post ablation. The pin recordings are stratified based on the percent reduction in the amplitude from pre- to post ablation. Level 1 corresponds to the maximal reduction likely to correspond to the pins that are in maximal contact with the tissues whereas level 4 represents the least reduction likely to represent the pin electrodes in poor or no contact with the tissues. 


\section{Panel A: Atrial tachycardia}

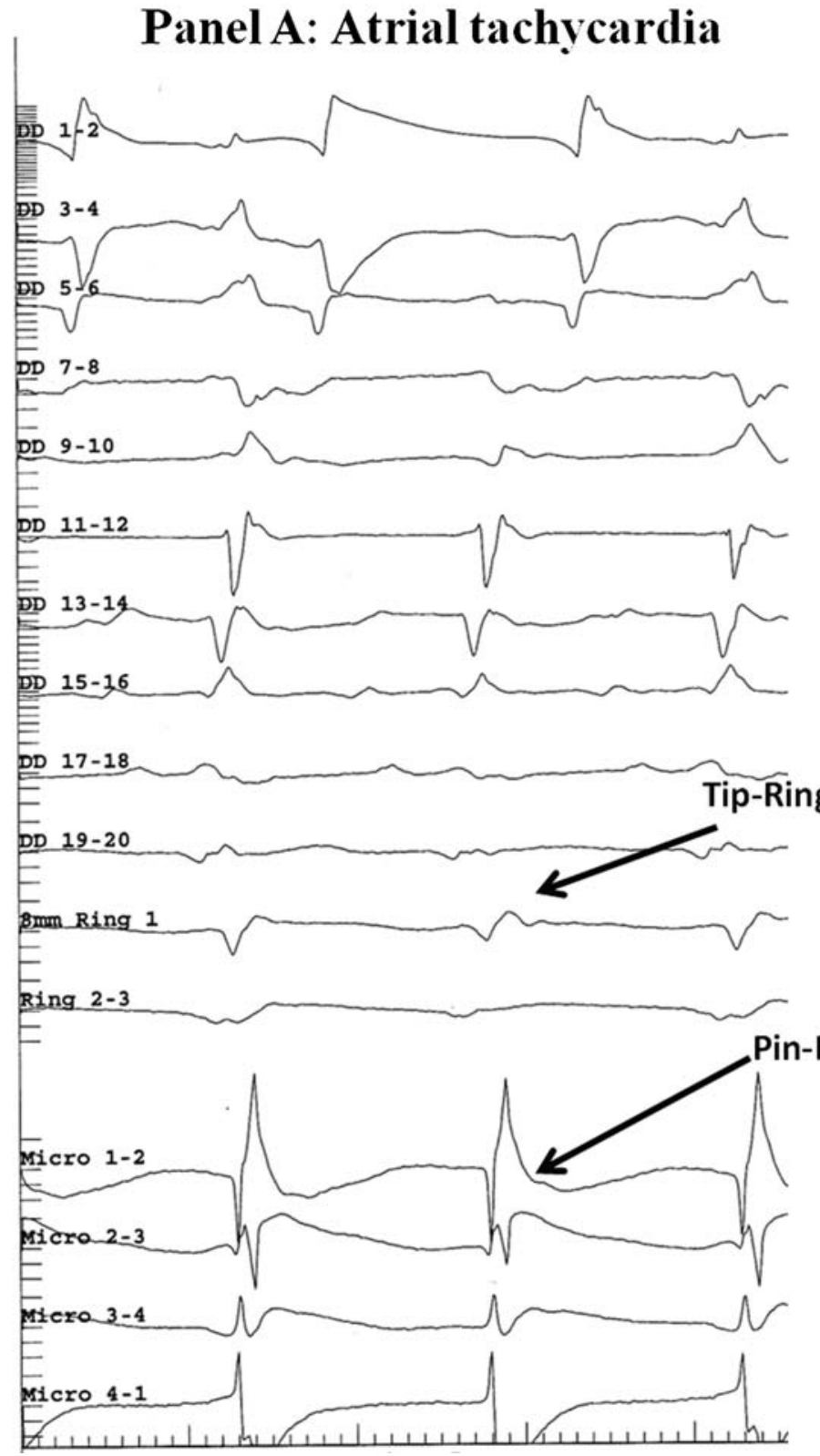

\section{Panel B: Conversion to SR during ablation}

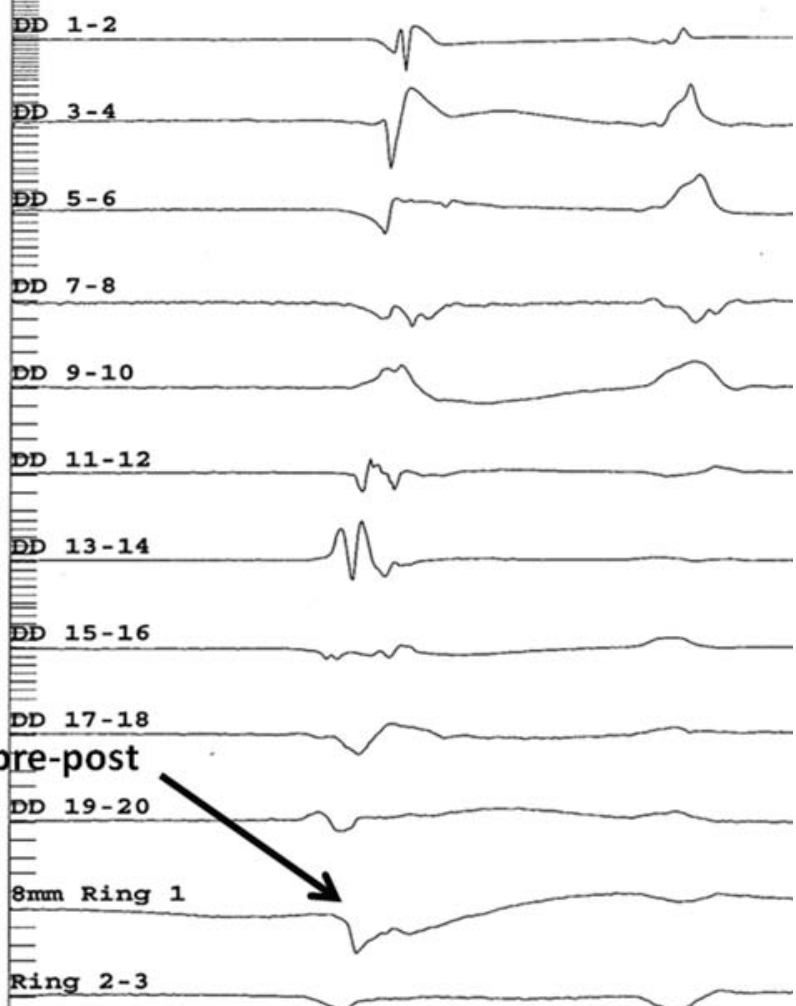

Ring 2-3

Figure 5: An example of the differences in the local electrogram recordings pre- and post ablation from the pin-pin versus the tip to the ring electrode is shown. The upper arrows point to the tip-ring and lower arrows to the pin-pin preand post ablation. (a) The atria are in tachycardia at a cycle length of $320 \mathrm{~ms}$, which terminated to normal sinus rhythm during the ablation of a gap in the intracaval line. The pre-ablation electrograms recorded from the tip-ring (upper left arrow) point to a low amplitude low frequency local electrogram recording. In contrast the pin-pin recordings from pin 1-2 (top tracing left lower arrow) exhibit high amplitude high maximum frequency pre-ablation with elimination of that signal and conversion of the atrial tachycardia during the application of the radiofrequency to these tissues. While marked changes are noted in the pin-pin, minimal changes are noted in the tip-ring recordings. The pin electrodes were much more successful in identifying viable tissue responsible for the formation of gaps and the induction of atrial tachycardias.

lesions. This made correlation of lesion transmurality to absolute electrogram decrease of each individual ablation impossible to assess. However, post remapping and ablation of the gaps no sustainable arrhythmias could be induced at the completion of the studies indicative of successful transmural linear lesion formation. This was confirmed with conduction block and tetrazolium staining. The difference in electrogram amplitude stratified by
PE recordings provides a potentially useful means of assessing contact.

\section{Conclusions}

Using this novel new catheter technology, monitoring the amplitude and maximum frequency as recorded by the 


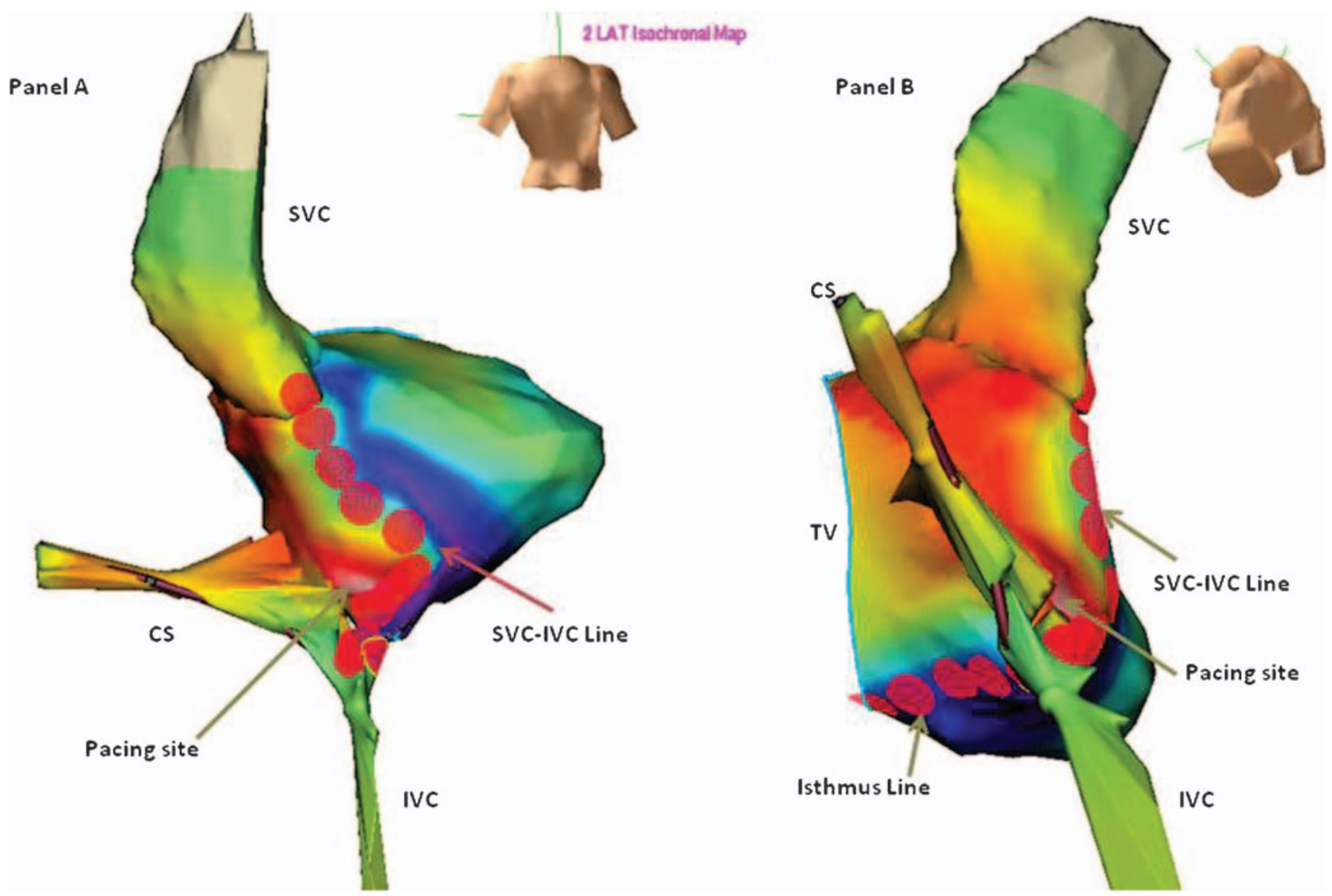

Figure 6: Post inferior vena cava and superior vena cava and isthmus linear lesions while pacing from the right atrial posterior lateral wall. The NaVx map shows distinct activation pattern confirming conduction block. SVC - Superior Vena Cava, IVC Inferior Vena Cava, CS - Coronary Sinus, TV - Tricuspid valve.

PEs are indicators of effective lesion creation. Utilizing the pin to pin recordings, the enhanced electrophysiological discrimination of viable versus ablated tissues is a novel and useful tool to identify linear lesions contiguity gaps that often cause atrial tachycardia. Additionally, the use of pin recordings has the potential of increasing the safety and reduces the number of RF applications by limiting the RFA applications to only viable tissues. Despite the addition of the PEs, catheter performance and steerability remained intact.

More importantly, whether it is $8 \mathrm{~mm}(12 \mathrm{~mm}$ recording distance) or $4 \mathrm{~mm}$ ( $9 \mathrm{~mm}$ recording distance) this investigation for the first time defines the utility of monitoring the localized recording that is limited to the ablated tissue to define the efficacy of the ablation both in the amplitude and frequency domain. Both the reduction

\section{Panel A}

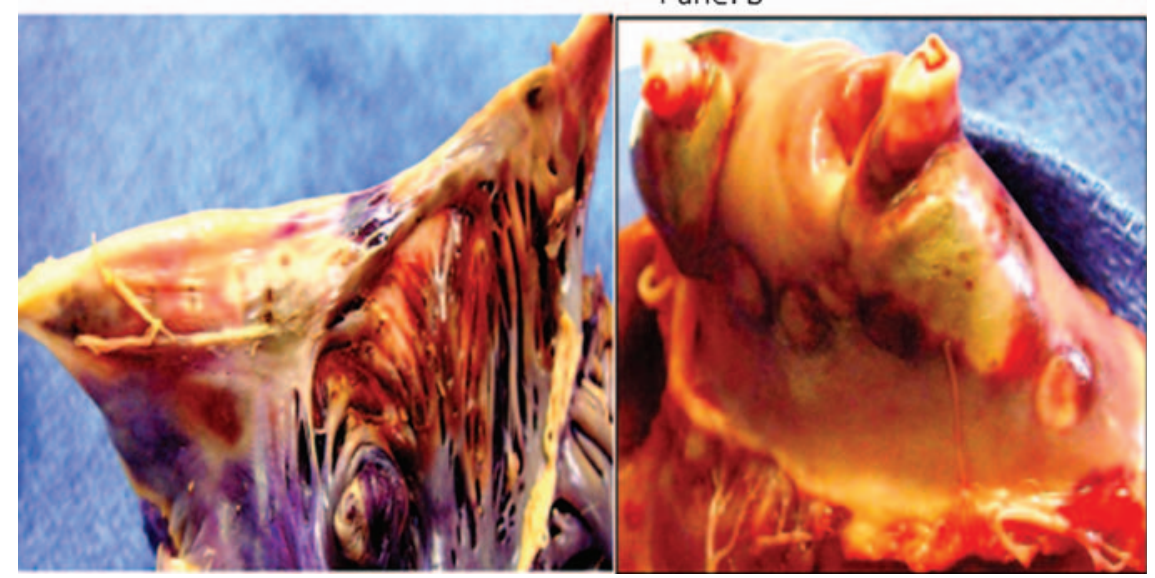

Figure 7: The endocardial surfaces on the inverted right and left atria. (A) The lesions in the right atria and (B) the left atria. No catheter char was identified during the course of ablation and the tetrazolium-stained lesion confirmed a contiguous transmural lesion. 
of the local electrogram amplitude and the frequency shift are uniquely associated with atrial lesion formation while recording from the PEs. These changes were associated with lesion formation and a surrogate of tissue contact.

\section{References}

1. Petersen HH, Chen PX, Svendsen A, Haunsø JH. Lesion dimensions during temperature-controlled radiofrequency catheter ablation of left ventricular porcine myocardium: impact of ablation site, electrode size, and convective cooling. Circulation 1999; 19; 99:319-325.

2. Nakagawa H, Yamanashi WS, Pitha JV, et al. Comparison of in vivo tissue temperature profile and lesion geometry for radiofrequency ablation with a saline-irrigated electrode versus temperature control in a canine thigh muscle preparation. Circulation 1995; 91:2264-2273.

3. Demazumder D, Mirotznik MS, Schwartzman D. Biophysics of radiofrequency ablation using an irrigated electrode. J Interven Cardiac Electrophysiol 2001; 5:377-389.

4. Avitall B, Helms R, Koblish J, et al. The creation of linear contiguous lesions in the atria with an expandable loop catheter. J Am Coll Cardiol 1999; 15:972-984.

5. Sanchez JE, Kay NG, Benser ME, et al. Identification of Transmural Necrosis Along a Linear catheter ablation lesion during atrial fibrillation and sinus rhythm. J Interven Cardiac Electrophysiol 2003; 8:9-17.

6. Jumrussirikul P, Atiga WL, Lardo A, et al. prospective comparison of lesions created using a multipolar microcatheter ablation system with those created using a pullback approach with standard radiofrequency ablation in the canine atrium. FACE 2000; 23:203-213.
7. Van Rensburg H, Willems R, Holemans P, et al. Simultaneous creation and evaluation of linear radiofrequency lesions. J Interven Cardiac Electrophysiol 2002; 6:215-224.

8. Tsai CF, Tai CT, Yu WC, et al. Is 8-mm more effective than 4-mm tip electrode catheter for ablation of typical atrial flutter? Circulation 1999; 100:768-771.

9. Sjogren AL. Left ventricular wall thickness determined by ultrasound in 100 subjects without heart disease. Chest 1971; 60:341-346.

10. Villamizar NR, Crow JH, Piacentino V, et al. Reproducibility of left atrial ablation with high-intensity focused ultrasound energy in a calf model. J Thorac Cardiovasc Surg 2010; 140: 1381-1387.

11. Scanavacca MI, D'Ávila A, Parga J, et al. Left atrialesophageal fistula following radiofrequency catheter ablation of atrial fibrillation. J Cardiovasc Electrophysiol 2004; 15: 960-962.

12. Pappone C, Oral H, Santinelli V, et al. Atrio-esophageal fistula as a complication of percutaneous transcatheter ablation of atrial fibrillation. Circulation 2004; 109:27242726.

13. Bunch TJ, Bruce KG, Johnson SB, et al. Analysis of cathetertip (8-mm) and actual tissue temperatures achieved during radiofrequency ablation at the orifice of the pulmonary vein. Circulation 2004; 110:2988-2995.

14. Berjano JE, Fernando H. Esophageal temperature during radiofrequency-catheter ablation of left atrium: a threedimensional computer modeling study. J Cardiovasc Electrophysiol 2006:17:405-410.

15. Hummel JD, Daoud EG, Joseph M, et al. Ablation tips with embedded microelectrodes provide enhanced spatial resolution in a canine model. Heart Rhythm. 2010:7:5: S211. 